\title{
Identidad y subjetivación política en el Movimiento por la salud digna en Chiloé
}

\author{
Nayadeth Arriagada \\ Universidad Alberto Hurtado, Santiago de Chile. \\ E-mail: narriaga@alumnos.uahurtado.cl
}

\begin{abstract}
Resumen $^{\mathbf{1}}$ : El presente artículo tiene como fin describir la relación entre los procesos de subjetivación política y construcción de identidad, que experimentan aquellas personas que participan del movimiento por la salud digna en Chiloé el 2013. Los resultados muestran que los procesos de subjetivación están íntimamente relacionados con la identidad de estas personas, en la medida en que la identidad se experimenta como el movimiento entre una afirmación negativa: la experiencia nostálgica por un pasado perdido -asociado a valores compartidos-, y por una afirmación positiva: el compromiso íntimamente ligado al deseo de ser actor colectivo, propio de la subjetivación-. Pero además es posible establecer que la subjetivación es un proceso político de desidentificación y consiguiente reidentificación, dado por entender que el problema de la salud no es propio del archipiélago, ni tampoco individual, sino que colectivo y generalizado a nivel país, asociado a un adversario político: Estado chileno.
\end{abstract}

Palabras clave: Movimientos sociales, subjetividad, subjetivación política, identidad, territorio.

\section{Identity and political subjectivity in the movement for dignified health in Chiloé}

\begin{abstract}
This article aims to describe the connection between political subjectivization and construction of identity of the people involved in the "movimiento por la salud digna" (movement for dignified health) in Chiloé, occurred in 2013. The results show that the subjectivization processes are closely linked with the identity of these people, to the extent that identity is experienced as the movement between a negative assertion: the nostalgic experience for a lost past -related to shared values- and a positive assertion: the commitment -closely associated to the desire of being an actor, that characterizes subjectivization-. Additionally it is possible to establish that subjectivization is a political exercise of de-identification and subsequent reidentification, due to the understanding that the health problem is not an individual issue, nor that it occurs only in this particular territory. On the contrary, that it is a collective and generalized problem in the whole country, connected to a political adversary: the Chilean State.
\end{abstract}

Keywords: Social movements, subjectivity, political subjectivization, identity, territory. 


\section{Identidade e subjetivação política no Movimento pela saúde digna em Chiloé}

Resumo: O presente artigo tem como finalidade descrever a relação entre os processos de subjetivação política e construção de identidade, que experimentam aquelas pessoas que participam do movimento pela saúde digna no Chiloé durante o ano 2013. Os resultados mostram que os processos de subjetivação estão intimamente relacionados com a identidade destas pessoas, na medida em que a identidade se experimenta como o movimento entre uma afirmação negativa: a experiência nostálgica por um passado perdido -associado a valores compartilhados, e por uma afirmação positiva: o compromisso -intimamente ligado ao desejo de ser ator coletivo, próprio da subjetivação-. Porém, além disso é possível estabelecer que a subjetivação é um processo político de desidentificação e conseguinte reidentificação, por entender que o problema da saúde não é próprio do arquipélago, nem tampouco individual, mas coletivo e generalizado ao nível do país, associado a um adversário político: O Estado chileno.

Palavras-chave: Movimentos sociais, subjetividade, subjetivação política, identidade, território.

$* * *$

\section{Introducción}

Desde el año 2011 a la fecha, se puede constatar en Chile la creciente presencia de la protesta social y de diversas movilizaciones. Las cifras señalan que el periodo del 2011 se posiciona como el periodo con mayor nivel de movilización social desde los años noventa (Segovia \& Gamboa 2012). En este contexto, es el movimiento estudiantil de ese año el que parecía marcar un hito sin precedentes. Con demandas por una educación pública, gratuita, de calidad y en contra del lucro, no sólo llama la atención por apelar a reformas más estructurales y por la continuidad que posee como movimiento, sino por el carácter de ruptura que genera en un Chile que en veinte años de post-dictadura, no había presenciado movimientos sociales con tanta adhesión desde la ciudadanía.

En esta línea, la pregunta por describir y explicar el surgimiento de tal movimiento toma distintos matices desde la sociología. Una serie de trabajos, desde la perspectiva de los movimientos sociales, buscan describir al movimiento estudiantil en su aspecto más organizacional, es decir, en virtud de elementos como lo son los repertorios de acción que los estudiantes llevan a cabo, demandas, y el impacto del mismo (Garretón 2011; Fernández 2013, Urra 2012; Durán 2012). Otros autores, en cambio, tratarán de buscarle un sentido explicativo a dicho proceso desde el vínculo entre ciudadanía y sistema político. De este modo, autores hablan del surgimiento del movimiento estudiantil producto de una crisis de representatividad, gatillada por el ascenso de la derecha al poder luego de veinte años (Mira 2011). Autores como Fleet (2011) dirán que se trata, más bien, de una crisis de legitimidad, entendida como una fracción en el sistema de dominación en su conjunto, plasmado en las cifras que muestran la gran desafección de la 
ciudadanía con la institucionalidad política. Esto acompañado de una ampliación y diferenciación de grupos sociales que lleva consigo el surgimiento de una nueva clase media (Fleet 2011). En una línea argumentativa similar, Garretón también establecerá un vínculo entre movimientos sociales y sistema político, señalando que una de las principales consecuencias del orden sociopolítico heredado de la dictadura es la "[...] desarticulación de los actores clásicos de la matriz estatal nacional popular y el surgimiento de una diversidad de actores, más distantes o también marginales al sistema político partidario” (Garretón et. al. 2011: 123).

Otras explicaciones pondrán su centro en las consecuencias del modelo neo-liberal en la subjetividad de las personas (Mayol 2011; Núñez 2012; Azócar 2012). En esta línea, se encuentran perspectivas como la de Mayol (2011), quien propone que, desde los años noventa a la fecha, se forjó una subjetividad que toleró un creciente malestar basado en los desequilibrios sociales producidos por el modelo. Dicho malestar acumulado encontraría su cauce el año 2011 con el movimiento estudiantil que pone en cuestión no sólo el tema de la educación, sino que tematiza la forma en que en nuestro país se ha estructurado lo público y lo privado. Así, desde el 2011, se podría constatar la presencia de procesos de politización del malestar (Mayol\&Azócar 2011;Mayol A., Azócar, C. \& Brega, C. 2011). Autores como Carlos Azócar (2013) también señalarán que el surgimiento de este movimiento se debe a las consecuencias del modelo neoliberal, aunque, a diferencia de otros planteamientos, este autor señala que el movimiento estudiantil surge producto del "fraude" de la movilidad social ascendente que prometía el modelo de desarrollo económico, el cual encuentra en la educación su caso paradigmático, que llevan a una politización del malestar producto de la no realización del ideal liberal meritocrático (Azócar 2013).

Sin embargo, como señalamos anteriormente, desde ese año no sólo se advierte la presencia de un movimiento social por la educación, sino que el surgimiento de una serie de movilizaciones de tipo regional. Estas son inaugurados por la protesta en Magallanes en enero del 2011, producto del alza del combustible. Dicho conflicto,que se inició con la toma del aeropuerto de Punta Arenas el cinco de enero, incluyó también amplias movilizaciones sociales en la zona, el cierre de los accesos a Punta Arenas y de pasos fronterizos (Segovia \& Gamboa 2012). Similar a dicho estallido es lo que ocurre posteriormente en Aysén el año 2012, donde las demandas también apelan a una mayor autonomía de la zona.

Si bien las explicaciones para tales movilizaciones pueden ir en la línea de las explicaciones anteriores para el caso de la educación, dando cuenta de un fenómeno a nivel nacional y regional de malestar, donde se interpela al Estado por la forma en que se articula lo privado y lo público (Mayol 2011), o una crisis de legitimidad del sistema político y de la democracia incompleta con enclaves autoritarios (Fleet 2011; Garretón et al. 2011), o los efectos perversos del modelo económico continuado por los gobiernos de la Concertación² (Azócar 2012), cabe añadir también las propuestas 
de los estudios de descentralización, que pueden complementar las explicaciones recién expuestas.

A modo de contextualización histórica, cabe señalar que fue la dictadura militar la que promulgó una regionalización planificada en nuestro país, entregando mayores competencias a municipios, en contradicción con la fuerte represión ejercida hacia la ciudadanía. Este proceso sería "desde arriba”, imponiendo una división territorial que vino a menoscabar a las provincias (Valenzuela 1999: 105). En realidad, dicha regionalización respondería dos razones: a mejorar el desarrollo económico en virtud de los recursos naturales de cada región, así como una razón de carácter geopolítico: en un contexto de posible guerra con los países vecinos, era necesario generar un sistema de seguridad nacional y defensa de los contornos fronterizos en todas las regiones. (Valenzuela 1999; Montecinos 2005). Con la llegada de la democracia, los gobiernos de la Concertación siguieron profundizando los procesos de desconcentración administrativa, sin generar una real descentralización (Valenzuela 1995: 96).

De esta manera, Egon Montecinos (2013) argumenta que las movilizaciones a nivel regional como la de Magallanes y Aysén, tienen en su centro una crítica a la dimensión democrática del desarrollo regional. Es decir, dichas manifestaciones dan cuenta de un descontento patente a nivel nacional, pero, por sobre todo, un descontento a nivel local por el excesivo centralismo de carácter histórico en nuestro país. Así, sostiene como hipótesis que dichos problemas regionales tienen que ver con una falta de reconocimiento de las provincias como "sujetos políticos", así como la escasa transferencia y distribución de poder político administrativo y fiscal (descentralización) (Montecinos 2013: 2).

Es así como en este contexto de emergencia de la protesta social, y antecedido por los movimientos regionalistas recién mencionados, se levanta el 2013 un nuevo estallido de protesta social regional: el movimiento por la salud digna en Chiloé. A partir del fallecimiento negligente de dos personas en Quellón, surge la preocupación por las condiciones de precariedad del sistema de salud en que se encuentra la ciudad, y toda la isla. De esta manera, se levantan acciones colectivas donde incluso autoridades locales se hacen presentes, así como siete asambleas comunales, quienes redactan un petitorio provincial único con el fin de luchar por condiciones de salud pública digna para todo Chiloé. La protesta social culmina tras una serie de negociaciones y compromisos tomados entre el gobierno central con las autoridades locales. Sin embargo, las asambleas sociales por comuna siguen movilizándose de manera más pasiva, a la espera de una respuesta al petitorio provincial.

Si bien, cabe señalar que no abundan los estudios que den cuenta de tales conflictos de tipo regional -por su carácter reciente y alejado geográficamente- se deben destacar los aportes que puedan entregar los enfoques de descentralización para entender dichos movimientos, introduciendo importantes variables como es la demanda por autonomía y descen- 
tralización en la administración político-económica de nuestro país. Sin embargo, y en directa relación con los estudios sobre el movimiento estudiantil anteriormente mencionados, tales explicaciones sólo relevan los aspectos más estructurales del problema (trasfondo de las demandas, repertorios de acción, crisis de legitimidad del sistema, dislocación del modelo económico, falta de autonomía en provincias), dando cuenta de contextos que no necesariamente pueden desembocar en acción colectiva.

En este sentido, toma relevancia aportar al estudio de los movimientos sociales a nivel regional en Chile desde una mirada poco explorada: la experiencia de los protagonistas. La perspectiva que en este artículo se utilizará propone que los procesos de movilización social se pueden entender a partir de los procesos de subjetivación de los individuos, ya que los movimientos sociales son la estructuración de ese deseo de ser actor, dado por dicho proceso de subjetivación. Pues tal como señala Touraine "el sujeto es la voluntad de un individuo de obrar y ser reconocido como actor” (Touraine 1994: 207).

En virtud de ello, la presente investigación tiene como finalidad describir los procesos de subjetivación política que experimentan aquellas personas que participan en el movimiento por la salud digna en Chiloé el año 2013, en relación a la construcción de identidad personal. Se establece como hipótesis que la subjetivación política de estas personas se construye en relación a lo que se puede denominar un vínculo "dañado" entre los individuos de esta zona con el Estado a través de las políticas de salud, pero, a su vez, asociado a un fuerte componente identitario en términos de arraigo.

Así, el presente artículo se estructurade la siguiente manera: en primer lugar, abordaremos algunos antecedentes generales sobre Chiloé. Luego, daremos cuenta de los conceptos y la perspectiva teórica en que se basa esta investigación, la cual es profundizada en el análisis de resultados. La tercera parte aborda los aspectos metodológicos y en cuarto lugar, se exponen los principales resultados, profundizando, a su vez, en los conceptos en que esta investigación se basa. Finalmente, se esbozan las principales conclusiones y los desafíos a futuro.

\section{Antecedentes generales de Chiloé}

La provincia de Chiloé se encuentra a 1.200 kilómetros al sur de Santiago, perteneciente a la región de Los Lagos, y cuenta con una población de 185.445 personas $^{3}$.

Cabe señalar que Chiloé es un espacio altamente heterogéneo en términos de ingreso, pobreza y desigualdad. A grosso modo, se identifican tres zonas claramente diferenciadas: Norte, Centro y Sur de Chiloé, las cuales guardan estrecha relación con las dinámicas demográficas y económicas de cada una de estas tres zonas, y corresponden a las áreas de influen- 
cia de los tres núcleos urbanos: Ancud en el norte, Castro en el centro, y Quellón en el sur (Mondrego, F., Ramírez, E., Macé, J., Yáñez, R. 2009: 3).

Históricamente, Chiloé ha sido un territorio con demandas de distinta naturaleza hacia el Estado, como lo es la mejora de las condiciones de equipamiento y conectividad de la isla. Ésta última, acorde con Montecinos\& Neira (2013), se ha venido planteando hace varios años a través de la idea de la construcción de un puente en el canal de Chacao, la cual encuentra eco en los años noventa con el gobierno de Eduardo Frei Ruiz-Tagle. Años más tarde, Ricardo Lagos lo incluirá como uno de los proyectos del Bicentenario (Montecinos\& Neira 2013: 496). Dicha propuesta no estuvo exenta de críticas desde la comunidad, ya sea con argumentos técnicos respecto a su construcción, así como los posibles problemas que podría generar en la cultura de la propia isla. Quienes se encontraban a favor argumentaban que dicho proyecto permitiría una mejora en la calidad de vida de los habitantes, especialmente en cuanto a las posibilidades de acceder con mayor rapidez a mejores servicios de salud en el continente.

Con el primer gobierno de Michelle Bachelet, se cae la posibilidad de construir el proyecto, puesto que el costo de realización superaba los valores estimados. Dicho anuncio generó la molestia de la comunidad que se declaraba a favor del puente. Se realizaron diversas manifestaciones en la isla, incluyendo el aislamiento total de la zona por un día (ídem). A raíz de estos hechos, es que el gobierno de la entonces Presidenta Michelle Bachelet anuncia elPlan Chiloé, el cual tenía como finalidad el desarrollo de la isla a través de la mejora de una serie de políticas públicas de diversa índole, como lo son infraestructura, educación, salud y electrificación rural, en virtud de tareas pendientes del gobierno con el territorio, pero a la vez integrando demandas de la propia población (ídem).

Sin embargo, como las movilizaciones acaecidas en mayo del 2013 dejan entrever, todavía existen graves falencias en el sistema de salud de la isla, por lo que las promesas del Plan Chiloé aún no logran su total concreción.

\section{Conceptos centrales}

El modelo teórico en el que se basa la presente investigación, toma los planteamientos de Francois Dubet (1989, 2010), Alain Touraine (1994, 1995) y Jacques Rancière (2005) principalmente, para abordar las nociones de identidad y subjetivación política. Dichas conceptualizaciones son complementadas además con las propuestas de Paul Ricoeur (2005) sobre la idea de identidad, Judith Butler (2010) a la hora de definir la subjetivación.

\section{Identidad}

El problema de la identidad ha sido tratado desde distintas perspectivas en la sociología (Taylor 1997; Honneth 1997; Larraín 2001; De Singly\&Martuccelli 2012). Sin embargo, para efectos de este artículo, utili- 
zaremos los planteamientos de Francois Dubet a la hora de hablar de identidad, en la medida en que "[...] la construcción de la identidad social es inseparable de una concepción sociológica del sujeto” (Dubet 1989: 520).

En la conceptualización de lo que define como experiencia social, Dubet establece que a cada lógica de acción -integración, estrategia y subjetivación-, le corresponde una forma de identificación social: identidad por integración, identidad como recurso, e identidad como compromiso, respectivamente.

Las diferentes dimensiones de la identidad señaladas no tienen unidad propia, sino que se configuran con sus propias leyes. De esta manera, el autor señala que el interés de la sociología de la identidad es mostrar cómo estas distintas formas de definición de sí se logran articular. La identidad, por tanto, no es una dimensión estática ni unitaria, y tampoco se trata de una afirmación positiva. Como lo señala el mismo autor, "los actores dominados que construyen movimientos contestatarios [...] buscan en la cultura dominante los valores sobre los cuales fundamentar su identidad y de la que, al mismo tiempo, están privados: ciudadanos privados de derechos, trabajadores explotados. Hoy, la identidad movilizada es la del sujeto mismo, definida como la capacidad de ser actor” (Dubet 1989: 544).

\section{Subjetivación, lo político y subjetivación política}

Tanto Dubet (2010) como Touraine (1994) establecen que el estudio de la acción social es indisociable de la noción de sujeto, ya que la subjetivación no sería otra cosa que la estructuración del deseo de ser actor, condición de posibilidad de toda acción colectiva. No se trata, por tanto, de una afirmación positiva, sino que más bien de una afirmación negativa: es un cuestionamiento a patrones de integración de la sociedad, un malestar dado por un distanciamiento crítico de roles y de definiciones culturales que se imponen. En la denuncia de los contratiempos y obstáculos a la hora de realizar el propio proyecto, es que se manifiesta el deseo de ser actor. Pero la subjetivación es un proceso que no remite al individuo solamente, sino que también es colectivo. Como lo señala Angelcos (2010), si bien el deseo de ser actor aparece primero en términos individuales, bajo la forma de sufrimientos o desgarros de la identidad, la capacidad de transformación por parte de los actores sociales, depende de la capacidad de articularse (posición de sujeto) en torno a proyectos colectivos que desafíen orientaciones culturales hegemónicas (Angelcos 2010).

Esto último es analizado por Rancière (2006), quien define que lo político es el encuentro de dos lógicas heterogéneas: la policía-el gobierno, el orden de jerarquías y funciones- y la política, la cual se basa en la lógica de la emancipación. Como el mismo autorlo señala: "lo político es la escena donde la verificación de la igualdad debe tomar la forma del tratamiento de un daño. Por ende, lo político será el terreno del encuentro entre política y policía en el tratamiento de un daño” (Rancière, 2006: 18). Esta definición de lo político se relaciona con lo que Touraine (1995) señala a la 
hora de definir la naturaleza de los movimientos sociales en dos sentidos. En primer lugar, al igual que Rancière, la dimensión del conflicto es clave para el surgimiento de los movimientos y luchas sociales, dado por el principio de oposición -identificación de un adversario social-. Segundo, no basta con entender el carácter conflictivo de los movimientos, sino que también su capacidad de disputar cierto dominio, dado por el principio de totalidad.

Rancière además establece que la idea de política es indisociable de la noción de sujeto, en la medida en la lucha por disputar orientaciones culturales, los individuos se desprenden de un yo, de una identidad impuesta por una en construcción.

En este sentido, podemos ver que, al igual que Touraine y Dubet, Rancière establece que los procesos de subjetivación corresponden a una "teología negativa": el desprendimiento o distanciamiento crítico de cierta identidad impuesta, por la búsqueda de una propia resignificada en términos políticos. Así, los tres autores coincidirían en que, desde la lógica de la subjetivación, lo social se entiende como la inserción en relaciones de dominación, donde el vínculo con lo político estaría en cuestionar y disputar patrones de integración social.

El siguiente esquema resume el modelo teórico en que se basa el presente artículo, brevemente expuesto más arriba, y que será profundizado en el análisis de resultados:

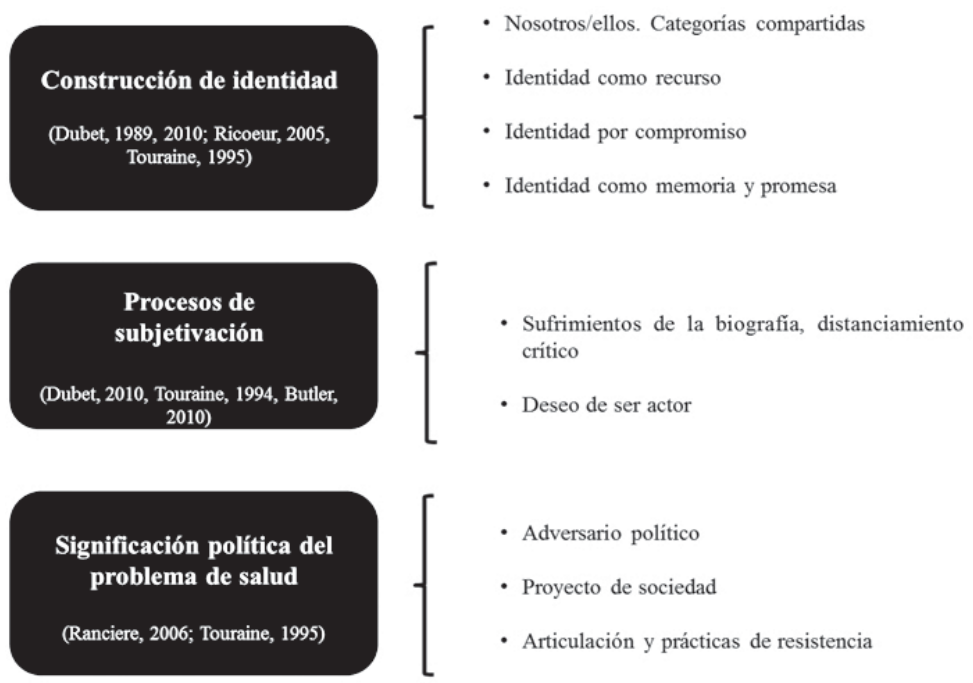

Esquema 1: Resumen conceptualización

Fuente: Elaboración propia 


\section{Metodología}

Para el desarrollo del presente artículo, se realizaron siete entrevistas semi-estructuradas basadas en el enfoque biográfico, a participantes de las asambleas sociales por la salud en Chiloé, específicamente de las ciudades de Castro y Ancud. El trabajo de campo fue realizado entre los meses de septiembre y octubre del 2013.

Cabe mencionar que los entrevistados fueron seleccionados por muestreo tipo bola de nieve, y sus nombres fueron reemplazados con el fin de resguardar la confidencialidad de la información.

\section{Análisis de resultados}

\section{Contenidos de la identidad}

Dubet (1989, 2010) establece que las distintas lógicas de acción social están ligadas a distintas formas de identidad. Cuando el autor habla de identidad por integración, señala que la identidad del actor es el correlato subjetivo de su integración al sistema, es decir, "no es sino la manera a través de la que el actor interioriza los valores institucionales por medio de roles” (Dubet 2010: 103). A este nivel, la sociedad es concebida como una organización de los roles orientados a valores colectivos, donde la acción social se adecúa a ello. La cultura, por tanto, será entendida como el edificio de valores comunes que sostienen la identidad. Así, individuo se define en virtud de su pertenencia, como una ascription, en torno a lo cual se estructura la distinción nosotros/ellos, es decir,una afirmación positiva de la personalidad social en oposición y distanciamiento del otro.

En este sentido, se puede constatar, en los discursos de los entrevistados, la presencia de una identidad por integración, en virtud de los valores compartidos que asocian al ser chilote: un determinado vínculo social basado en la solidaridad y cooperativismo de la comunidad. Sin embargo, esta identidad es vivenciada en términos de una nostalgia, pues hoy en día, la vida social chilota parece ir perdiendo dichos valores, primando cada vez más el individualismo. Llama la atención que dicha experiencia nostálgica de añoranza de un pasado comunitario que se está perdiendo en Chiloé, no reconoce diferencias etarias, ya que tal discurso es recurrente tanto en las personas más jóvenes de las asambleas como las mayores. Como lo señala Irma.:

“[...] Entonces yo vengo un poco de eso, de esa cultura de la Minga ${ }^{4}$, del ayudar al otro, y...claro después cuando me avecindé en la ciudad digamos, eh ya uno toma otro ritmo, se empapa un poco de esto de la ciudad, entonces en relación con los movimientos sociales, claro yo vengo con esa formación solidaria de que estos bien todos en comunidad, que no solamente yo pueda hacer mis cosas, realizarme como persona, sino que tenga la posibilidad el vecino de al la’o” (Entrevista Irma). 
Pero las asambleas sociales no sólo están conformadas por personas provenientes de Chiloé, sino que se puede constatar la presencia de personas provenientes de otros lugares del país, principalmente de Santiago, que años atrás migraron a la isla en búsqueda de nuevas oportunidades. En este sentido, cabe señalar que esta vivencia nostálgica por un pasado asociado a la vida en comunidad es compartido por estas personas que tienen experiencias de vida vinculadas a las poblaciones populares del gran Santiago décadas atrás, en donde la vida de barrio y el sentido de pertenencia al lugar, eran categorías compartidas de la identidad, y que se han ido perdiendo. Así, estas personas encuentran en Chiloé dicha forma de vida como un pasado más reciente y que aún tiene posibilidades de rescatarse. Tal como lo señala el entrevistado Carlos:

“[...] A veces añoro esa vida de barrio...eh que en esos años estaba muy teñida de lo que era la vida comunitaria. O sea, cuando murió mi padre me junté con los vecinos y los vecinos me decían....nosotros ayudamos a construir a tu papá la casa, y después le fuimos a construir el techo de la señora de allá, y después...o sea, una vida comunitaria súper rica y uno notaba que los vecinos habían estado en la construcción de mi casa, que mi papá había estado en la construcción de las otras casas, eh...y era una vida súper en común” (Entrevista Carlos).

Sin embargo, la identidad personal y colectiva de las personas que participan de las asambleas por la salud digna en Chiloé no sólo está asociada a una experiencia afectiva en términos de nostalgia, sino que también llama la atención la presencia de una estrategia para la acción en la apelación a lo local y lo propio. Es decir, varios de los entrevistados señalan que sus demandas se dirigen a lo local en primera instancia debido a que es más fácil convocar y movilizar al resto de las personas cuando se apunta a problemas que sienten más cercanos. Este elemento es el que Dubet relaciona al hablar de identidad como recurso, inserta en la lógica de acción por estrategia. Los individuos se definen por su posición relativa en el espacio en términos de status, donde la identidad puede ser entendida como un recurso para la acción definida en términos competitivos (Dubet 2010).

Pero no todo es estrategia: en los discursos de los entrevistados también es posible dar cuenta de un compromiso con la isla, con el cooperativismo al que apelan, y el territorio, que se expresa en el deseo de defender y recuperar lo propio que los caracteriza. Siguiendo con los planteamientos de Dubet, la identidad no sólo puede ser entendida por su sentido de pertenencia y como recurso, sino que también en términos de las convicciones de los actores. Así, Dubet establece finalmente que, al nivel de la acción social por subjetivación, nos encontramos con la identidad por compromiso, la cual significa definir la identidad como un compromiso con modelos y orientaciones culturales, así como los proyectos que permiten definir los intereses y superarlos (Dubet 2010). "Este compromiso con una representación cultural del sujeto, se vive como algo inacabado, como una "pasión imposible" y deseada que le permite definirse como autor de su propia vida, aunque eso sea a través del sufrimiento generado por la impo- 
sibilidad de realizar plenamente su proyecto” (Dubet 2010: 116).

En este sentido, la vivencia nostálgica de una vida comunitaria que se ha ido perdiendo, a su vez toma la forma de un compromiso para con la isla en términos identitarios. El deseo de recuperar el vínculo social que los entrevistados asocian a Chiloé es recurrente en sus discursos, y no sólo en las personas que pertenecen a la isla, sino que también es recurrente en aquellas personas que no son de Chiloé. Sumado a ello, la vida en naturaleza, ligada al campo, el mar y los bosques genera una convicción de la necesidad de defender el territorio, ante la amenaza del neoliberalismo a través de la tala de bosques por empresas forestales, el problema de las empresas salmoneras que ensucian los mares, y otras formas de explotación de los recursos naturales de la zona. Tal como lo señala una entrevistada:

“[...] Y entonces a los chilotes les pasan cosas, les quitan el agua, les van a colocar un puente, les quitan los bosques. No sé po, con mayor razón aún me gustaría quedarme para ayudar a que si van a haber cambios...que tienen que haberlos porque a veces uno se queda como con esa idea nostálgica de vivir en un lugar y uno tiene que aprender a desprenderse de eso porque las cosas cambian, pero que sea una determinación de la gente de acá porque no me gustan un montón de cosas que están sucediendo. Si po, si po. Yo siento que tengo una responsabilidad, cachai...con el lugar en que crecí” (Entrevista Gabriela).

Lo anterior, siguiendo los planteamientos de Paul Ricœur (2005), nos da cuenta de una identidad inserta en el tiempo, en base al reconocimiento de sí a partir del acto de hacer memoria y prometer. Ricœur señala que "la fenomenología de la promesa se concentra precisamente en el actor por el que el sí se compromete efectivamente” (Ricœur 2005: 135). En este sentido, la identidad de las personas entrevistadas, más que asociarse a una identidad estática, se vincula más con una afirmación positiva a un pasado integrador, un presente entendido en términos negativos dado por una vivencia nostálgica, y un futuro como compromiso y promesa, vinculado a un proyecto que defiende lo local.

Esta identidad por compromiso es similar a lo planteado por Vicente Espinoza (1993) en sus estudios sobre la identidad y lógicas de acción de los pobladores a fines de los ochenta. Este autor identifica cuatro lógicas de acción, donde la que denomina "lógica de defensa comunitaria" se caracterizaría por definir a los pobladores en términos no clasistas. "Aun cuando puede aparecer el deterioro de la confianza en los mecanismos de integración, ella da origen a una orientación de tipo comunitaria. En una comunidad todos tienen cabida; los une el ser los desheredados y la voluntad de vivir un orden solidario" (Espinoza 1993: 6).

\section{Procesos de subjetivación}

Como se mencionó anteriormente, la subjetivación es entendida como aquel proceso en que los individuos se distancian críticamente de roles o 
status impuestos, a partir de sufrimientos o desgarros de la experiencia. Esto, señala Dubet (2010), se debe a que los actores viven con más facilidad su actividad como sujetos en el sufrimiento que en la alegría, y el deseo de ser actor es más un proyecto ético que una realización (Dubet 2010). “[... ] Es en la actividad crítica que el individuo es invitado a desprenderse de sí, a transformarse en un filósofo” (íbid: 119). De esta manera, el sufrimiento o desgarro que genera en el individuo la imposibilidad de realizar su proyecto, permite que se pueda percibir como autor de su propia biografía.

En este sentido, en los discursos analizados, es posible identificar una serie de desgarros comunes en las biografías de las personas que participan del movimiento. En primer lugar, todos conocen el sistema de salud pública desde cerca, ya sea porque son usuarios, o porque tienen vínculos cercanos con profesionales de la salud. En el caso de los primeros, la constante espera y la impotencia son sentimientos que acompañan la experiencia, dada por un sistema de salud pública considerado como insuficiente:

“[...] Me tengo que levantar a las seis de la mañana para ir a pedir hora al médico, o he ido y no he podido conseguir hora. O he ido a urgencia a pegarme un plantón de cinco, seis horas. O sea, conozco el tema porque soy usuario, no estoy hablando de salud pública y me atiendo en una clínica” (Entrevista Francisco).

Este último elemento llama la atención en los relatos de los entrevistados: quienes son usuarios del sistema de salud, realzan esa vivencia en primera persona como un valor moral en el proceso de lucha por una salud digna. Pero, en el caso de algunas personas, esa vivencia en primera persona de las deficiencias del sistema de salud, toma la forma de una historia de fuertes desgarros. Tal es el caso de Carlos, quien vive con una inmovilidad irreversible en el pie producto de ello:

“[... el el año 2009, tuve un accidente aquí en Castro, en el verano. Nos desbarrancamos en el auto con mi pareja. [...] Yo salgo del auto, piso y se me da vuelta el pie. Y siento...llega la ambulancia al rato, me trasladan al hospital, eh...el paramédico me dice "lo suyo es grave...tiene una fractura de tibia y peroné”, los tienes quebrados y hay que operarlo urgente. Pero vuelva el lunes porque no hay traumatólogo. Ya, okei, me pusieron un yeso provisorio, tenía una herida también, y me voy a la casa, me voy a la casa. Con mucho dolor, un par de anti-inflamatorios, y voy el lunes. [...] Llego y me atiende el doctor y me dice...lo tuyo es grave, hay que operar urgente, pero no tengo cama. Vuelve el miércoles...vuelvo el miércoles, me vuelve a decir lo mismo...lo tuyo es grave pero no hay cama, vuelve el lunes. Y así pasan 15 días. [...] Consiguieron cama, y cuando me descubren ya el yeso me dicen...tu pie se instaló de una manera que no es apropiada, se puso mal y se apretó. Entonces lo que hay que hacer es destrabarlo, es un procedimiento muy complejo. No logró hacerlo, no...logré mejorar la lesión del pie, además cuando me habían puesto el yeso me habían dejado una herida, una herida que fue creciendo por la humedad. Perdí... un trozo de piel. Y perdí la movilidad en el pie derecho, el pie no me queda derecho, me queda con una 
inflamación que los médicos le llaman el pie equino, y perdí movilidad porque cuando debieron haberme operado no estuvo" (Entrevista Carlos)

En esta experiencia de desgarro, y en otras relatadas por los entrevistados, llama la atención la distinción que realizan entre las carencias objetivas del sistema y la voluntad de los médicos. Todos reconocen que no se trata de un mal trato de los profesionales de la salud, es más, destacan en ellos los esfuerzos y buena voluntad en la mayoría de los casos, sino que el problema remite más a carencias de tipo objetivas y materiales del sistema: falta de recursos, de especialistas, de consultorios para la totalidad de la población. En este sentido, se puede apreciar una crítica respecto a cómo el Estado de Chile operacionaliza las necesidades de la población respecto al tema de salud. Entrevistados se manifiestan críticos a los criterios de rentabilidad social que imperan en las políticas públicas y su aplicación, que no condice con las necesidades reales del archipiélago. Por tanto, la subjetivación está posibilitada por un distanciamiento crítico no respecto a roles, sino que a necesidades que se imponen y que no logran tener correspondencia con la realidad que viven las personas del territorio.

Por otro lado, también es posible identificar ciertos desgarros de la experiencia pero no por vivencias propias, sino que producto de sufrimientos de un otro, que generan en los entrevistados una afección tal como si fueran desgarros propios. En este sentido, es posible identificar historias de dolor asociadas al mal funcionamiento de salud, que no necesariamente corresponden a historias de personas cercanas. Es más, esas historias muchas veces no refieren a un "nombre propio", sino que se tratan de relatos que por la impotencia que generan, son recordados por los entrevistados: personas que por un mal diagnóstico o por las distancias de horas para llegar a un consultorio cercano, fallecen. Tal como lo expresa Vicente:

“[...] Ocurrió un hecho lamentable que fue la muerte de una niña embarazada, en el proceso de traslado a Castro y traslado a Puerto Montt. Y eso fue un cambio de lógica en la asamblea porque sentíamos ya no que estábamos en una marcha sino que estábamos en un funeral. Y eso fue lo más...por lo menos pa’ mí fue súper fuerte” (Entrevista a Vicente).

Este último elemento se relaciona con los planteamientos de Judith Butler (2010). Esta autora plantea que la visibilización de imágenes de guerra y de sus víctimas, debe desembocar en una apertura afectiva en términos de indignación, que nos permita cuestionar aquello que se entiende como vida, y así, derivar en una "ética del cuidado". En este sentido, la afección por el sufrimiento de otro toma centralidad, tal como la autora señala: “¿Cuál es nuestra responsabilidad hacia quienes no conocemos, hacia quienes parecen poner a prueba nuestro sentido de pertenecer o desafiar las normas del parecido al uso?” (Butler 2010: 61). Así, plantea cuestionar los marcos en que entendemos el "nosotros", planteando un "nosotros" de tipo global que haga frente a políticas de imposición, que 
generan una distribución desigual del duelo público. Similar a lo que plantea Luc Boltanski (1999), en el estudio de las implicaciones morales y políticas del "sufrimiento a distancia" en un espectador a través de los medios.

En este sentido, los desgarros de la biografía generados por el sufrimiento de otro, tal como lo expresan los relatos de los entrevistados, parece clave para entender cómo se configura la subjetividad de estas personas. Los sufrimientos que expresan no son necesariamente en primera persona, sino que muchas veces están referidas a experiencias ajenas, que generan en el "yo"una indignación, tal como lo expresa Butler o Boltanski.

Pero también llama la atención la existencia de desgarros en torno a la participación política de tipo institucional. En las biografías de las personas que participan en el movimiento, es posible identificar cierta trayectoria asociada a partidos políticos, u otro tipo de organizaciones políticas, pero que en determinado momento decepcionaron a estas personas. Así, es posible identificar desgarros de la identidad dado por una desilusión de la política partidista.

Esto último caracteriza otro elemento que compone la subjetividad: el deseo de ser actor. Como señalamos anteriormente, la subjetivación consiste precisamente en el deseo de ser actor de la propia vida, ante el sufrimiento generado por desgarros de la biografía (Dubet 2010). En virtud de ello, es posible dilucidar en los entrevistados un deseo de ser actor, pero, producto del distanciamiento crítico respecto a la política institucional, se trataría de un determinado tipo de actor: actor colectivo. Los entrevistados no asocian su proyecto con la política partidista, sino que explicitan un deseo de ser un actor de la calle, de los movimientos sociales. Tal como lo expresa Carlos:

“[...] Eso me llama poderosamente la atención, eso me conmueve, que la sociedad hoy en día sea capaz de decir...no pasa nada con la dirigencia tradicional, no pasa nada con los partidos políticos, ni con diputados y senadores, y deciden bypassearlo y entablar una mesa de diálogo con el ministro. Eso me parece a mí que es lo que hay que hacer” (Entrevista Carlos).

Así, los procesos de subjetivación que experimentan las personas que participan en el movimiento por la salud, se caracterizarían por una serie de desgarros respecto a un sistema de salud defectuoso y precario, que no responde a las necesidades mínimas de una población. Estos sufrimientos pueden ser en primera persona, así como dados por la experiencia de un otro que los afecta de igual manera. A su vez, es posible identificar ciertos desgarros asociados a la política institucional, vivenciada en forma de desilusión con los partidos y ciertas lógicas de la política convencional. Todo esto deriva en un deseo de ser un tipo de actor por parte de los entrevistados: un actor colectivo. 


\section{Significaciones políticas del problema de la salud}

Sin embargo, a pesar de que las personas entrevistadas expresen un distanciamiento crítico respecto a la política institucional, sus discursos y acciones están lejos de tomar distancia con lo político. Así, es posible dilucidar la identificación explícita de un adversario político: el Estado de Chile, que avala el modelo neoliberal. Los entrevistados consideran que las políticas públicas en nuestro país carecen de una visión de territorio, donde además imperan lógicas de mercado en ámbitos que deberían ser considerados derechos universales, como es el caso de la salud. Pero también estas personas identifican el problema de la educación como otro eje deficiente a nivel territorial. En este sentido, llama la atención un elemento: los entrevistados consideran que el gobierno local es parte del adversario político al que se enfrentan, en la medida en que, conociendo todas las falencias del territorio, no visibilizó los problemas de la zona ante un gobierno central. Tal como lo expresan Mariana y Carlos:

“[...] Nuestras demandas van dirigidas a las dos formas, al gobierno local y al Estado, porque hay cosas que pasan por el Estado, y hay cosas que en realidad son una decisión directa del gobierno local” (Entrevista Mariana).

“[...] Yo creo que esto se dirige contra el Estado de Chile. Y la principal demanda que nosotros tenemos es que el Estado de Chile garantice el derecho humano a la salud. Sea para los habitantes de Castro, los habitantes de Chelín, de cualquier isla, que el Estado garantice ese derecho humano que está garantizado desde el año ‘ 45 por la convención internacional. $\mathrm{O}$ sea que si una persona se enferma, tenga derecho a acceder a la salud, tenga derecho a ser atendido, a no morir esperando y a no morir por una negligencia, como ha ocurrido. Entonces ¿Quiénes son los responsables de esto? El Estado de Chile, y cuando hablo del Estado de Chile, hablo desde la presidencia, los ministerios, los municipios que forman parte del Estado, los gobernadores, los intendentes, que todos se han advertido, todo han visto lo que pasa en Chiloé pero no hacen nada, son indolentes respecto al tema” (Entrevista Carlos).

Como mencionamos anteriormente, la dimensión adversarial ${ }^{5}$ es un elemento clave a la hora de entender lo político acorde con Touraine (1995) y Rancière (2006). Tal como lo define Touraine: "sólo se puede hablar de principio de oposición si el actor se siente enfrentado con una fuerza social general en un combate que ponga en entredicho las orientaciones generales de la vida social ” (Touraine 1995: 251).

Cabe mencionar además que los entrevistados reconocen en el Estado un abandono histórico hacia el territorio. Es decir, la identificación del Estado como un principio de oposición no tiene un carácter contingente, sino que las demandas dirigidas al Estado tienen una connotación histórica y que hoy en día toma su punto más crítico en el tema de salud: 
“[...] Chiloé siempre estuvo abandonado por parte del Estado, si es cosa de ver que Chiloé se anexó al territorio el año 1826...y a la fuerza. A la fuerza porque Simón Bolívar le dijo a Freire que si ellos no anexaban Chiloé se lo tomaban ellos" (Entrevista Juan).

En este sentido, la demanda por salud digna toma un carácter particular en el movimiento: que se tome en cuenta la condición de insularidad de Chiloé. Ante una geografía adversa, las personas que participan en el movimiento consideran que el Estado chileno debe tomar en cuenta dicha condición en el diseño de políticas públicas de salud para la isla. Tal como lo señala Vicente:

“[...] Como nosotros le decíamos, cómo el per cápita en salud son 3.500 pesos por persona, más un índice que tiene que ver con la vulnerabilidad social, porcentaje de ruralidad y otros temas, como no contempla el aislamiento, la insularidad que tenemos nosotros. O sea no es lo mismo invertir el per cápita en La Reina que invertir el per cápita en Ancud, no es lo mismo porque no tenemos las mismas condiciones” (Entrevista Vicente).

Sin embargo, llama la atención la presencia de un proceso de desidentificación, propio de la subjetivación política acorde con Rancière (2006). Este autor señala que la subjetivación es "la formación de un uno que no es un sí, sino la relación de un sí con otro” (Rancière, 2006: 21).Es decir, la subjetivación política es un proceso de desidentificación o desclasificación, el rechazo a una identidad impuesta por una identidad en construcción o resignificada, a través de la puesta en práctica de la igualdad — tratamiento de un daño- por personas que están juntas y que por tanto están “entre”. En esta línea, en los discursos de los entrevistados es posible dilucidar dicho proceso de desidentificación, en la medida en que reconocen que el problema no es exclusivo de Chiloé, sino que se trataría de un problema estructural del diseño de las políticas públicas y del Estado centralista, donde los problemas de salud del archipiélago no distan de los problemas que otras zonas más alejadas del centro del país poseen. Tal como lo expresan Gabriela y Francisco:

“[...] Yo creo que es el mismo problema que hay en todas partes. $\mathrm{O}$ sea, si tú prendes la tele te das cuenta que...de hecho, hay hospitales y centros de salud en que la salud pública es peor...la otra vez yo miraba en la tele un caso, donde era, en Rancagua no sé dónde era, que el hospital tenía hoyos en el techo, y una estufa gas para calefaccionar un hospital enorme”. (Entrevista Gabriela)

“[...] No porque nosotros seamos Chiloé vivimos así, sino que aquí hay una lógica del Estado, por lo tanto, la gente que plantea que no...resolvamos lo que pasa en Chiloé y con eso estamos, nosotros decimos no, el problema de Chiloé es uno más dentro de una cadena” (Entrevista Francisco).

Este último aspecto es reforzado por la conexión que realizan con los 
movimientos de Aysén, Magallanes y otras zonas, donde consideran que las demandas que dichos movimientos levantan son prácticamente las mismas.

Pero la significación política del problema de la salud no sólo consiste en la identificación de un adversario político, sino que también está dotada de cierta orientación a un principio de totalidad ${ }^{6}$.Como mencionamos anteriormente, Touraine (1995) plantea que no basta con entender la dimensión conflictiva de los movimientos sociales, sino que además se debe comprenderla existencia de un interés por disputar cierto dominio. Tal como Rancière establece que la lógica de "la política" tiene que ver con la acción de irrumpir con el desorden, el sistema de jerarquías y funciones ("la policía”), Touraine señala que el principio de totalidad“no es sino el sistema de acción histórica cuyos adversarios, situados en la doble dialéctica de las clases sociales, se disputan el dominio” (íbid: 252). Es decir, es el intento de controlar y orientar agencias sociales. En este sentido, los movimientos no sólo se manifiestan como contrarios a ciertas orientaciones, sino que también levantan proyectos o ideas de sociedad en virtud de esta diferenciación.

En el caso del movimiento por la salud en Chiloé, es posible dar cuenta de ciertaidea de sociedad presente en los discursos de los entrevistados, que trascienden el problema de salud de la isla. Es decir, se advierte un proyecto que va más allá de ampliar los marcos de integración social, en este caso, respecto a cómo se entiende el problema de la salud, sino que se quiere romper en términos estructurales con ciertos patrones de integración. En este sentido, el proyecto de la mayoría de los entrevistados consiste en poder romper con lógicas neoliberales presentes en beneficios que deberían ser derechos garantizados universalmente, como es salud y educación. Pero además los entrevistados apelan a una justicia social más allá de la demanda por salud. En esta línea, la idea de democracia como proyecto también se puede advertir en los relatos analizados. Las personas que participan del movimiento por la salud manifiestan el deseo de una sociedad más democrática en términos participativos, donde las personas puedan incidir en el diseño de las políticas que se les imponen. Nociones como "empoderamiento" y "la toma de conciencia" son recurrentes en sus discursos como fines en sí mismos, donde la organización es entendida como una herramienta para dicho fin. Tal como lo expresa Vicente:

“[...] El fin quizá sea para algunos sea construir un partido, nosotros decimos un instrumento; algo que nos sirva como pueblo para llegar a un fin. Y ese fin es transformar la sociedad legal o también informalmente. Informalmente porque también los proyectos no solamente son en función de lo que está pasando en el Estado, sino que también hay que cambiar la cultura, la visión económica, hay que cambiar incluso las prácticas económicas, volver algunas atrás a prácticas más comunitarias, familiares, que tienen que fusionarse en un proyecto nacional. No en función de la industria, el desarrollo, dejar eso de lado. Entonces...estamos hablando de un instrumento político y social, o sea un instrumento que sirva para 
eh...disputar poder, pero también para construir poder, que la gente se empodere, como dice la palabra, de sus propias decisiones. Que sea capaz de defenderlas, que genere nuevas prácticas en la comunidad.” (Entrevista Vicente)

Por último, cabe mencionar que la significación política del problema de salud también alcanza una dimensión práctica, dada por formas de articulación y prácticas de resistencia que el movimiento lleva a cabo.

La necesidad de articulación es clave a la hora de entender el paso de sujetos a actores sociales (Angelcos 2010). En este sentido, los entrevistados identifican obstáculos en la articulación de un actor colectivo en torno al problema de salud, dado por el individualismo que el sistema con lógica neoliberal impone de manera creciente. Tal como lo expresa Carlos:

“[...] Lo más difícil que vemos es que todavía estamos teñidos por un manto de indiferencia, por un manto de individualismo donde cada uno de nosotros, cada uno de los habitantes de este archipiélago siente...que todavía eh...se puede salvar solo. Si se enferma puede ir al médico particular, es no comprender que aquí hay un problema que afecta a un colectivo humano y que no se resuelve solo. Yo creo que este proceso modernizador que hoy día Chiloé está viviendo nos hace creer que es posible avanzar solos. Yo creo que se va a profundizar más todavía...yo creo que la indiferencia nos vas a seguir ganando” (Entrevista Carlos).

Sin embargo, en virtud de dichos obstáculos, es posible dar cuenta del ejercicio de acciones de resistencia en dos direcciones. Una hacia afuera, en torno a la visibilización de las demandas a partir de acciones como manifestaciones, marchas, incluso la toma del Servicio de Salud en la ciudad de Castro, ocurrida en mayo del 2013 cuando se levanta la protesta en Quellón.

Dentro de estas acciones, llama la atención además la presencia de cierta vocación de poder llevada a la práctica, como es el caso de la asamblea de Ancud, donde se levantó una candidatura a diputado independiente para competir en las elecciones de ese año. Dicha acción puede ser entendida como la puesta en práctica de la verificación de la igualdad que acuña Rancière (2006), en la medida en que la lógica de la política busca disputar el centro político con el fin de subvertir el orden de jerarquías y funciones -la policía-, y demostrar que dicho orden no es más que arbitrario.

Pero también es posible identificar prácticas de resistencia de carácter más endógeno,es decir, llevadas a cabo al interior de la organización de manera de hacer frente alindividualismo recién mencionado. Acorde con los entrevistados, las asambleas en Chiloé se caracterizarían por intentar replicar ciertos vínculos comunitarios, valores dados por el cooperativismo y la solidaridad, que además implican la recuperación de ese pasado integrador de la identidad colectiva que se añora, como lo mencionamos al principio 
del análisis. No sólo se organizan en torno al problema de salud, sino que también se generan actividades a beneficio de compañeros o personas de la comunidad que necesiten de cierta ayuda.

De esta manera, el problema de salud alcanza una significación en términos políticos dado por las dimensiones del conflicto y oposición, el deseo de un proyecto de sociedad más allá del problema de salud, y por la articulación de sujetos y realización de prácticas de resistencia que hacen frente a los obstáculos que estas personas logran identificar. En este sentido, acorde con las personas entrevistadas, el movimiento por la salud en Chiloé no se trataría simplemente de una demanda por el reconocimiento dentro de ciertos patrones de integración impuestos desde arriba por el Estado, a través de las políticas públicas, sino que un cuestionamiento de ello, sumado al deseo colectivo de subvertir dichos patrones.

\section{Conclusiones}

A estas alturas, es necesario en primer lugar, recapitular los principales resultados presentados anteriormente. Cabe mencionar que las asociaciones que aquí se presentarán no son conclusiones sino que el inicio de otras investigaciones, pues se debe reconocer la necesidad de profundizar en ellas en trabajos futuros.

Como ya mencionamos, la identidad de las personas que participan del movimiento por la salud digna en Castro y Ancud, se caracterizaría por ser una identidad inserta en el tiempo en términos de memoria y promesa. El ejercicio de memoria se expresa en la asociación de la identidad chilota a lo que Dubet (2010) denomina identidad por integración: valores compartidos dados por el sentido de pertenencia, cooperativismo y vínculos comunitarios, vivenciado en términos nostálgicos por tratarse de un pasado que se ha perdido. Pero también es posible dilucidar una identidad como recurso, en la medida en que estas personas manifiestan apelar a la identidad chilota como un recurso para la acción colectiva y movilización por el tema de salud. En este sentido, la identidad no sería un contenido positivo y unitario, sino que una articulación de distintas lógicas, donde finalmente podemos encontrar además una identidad por compromiso: la presencia de un ejercicio de promesa de defender lo propio; las riquezas del territorio insular, así como los valores compartidos en pérdida.

Ligado a lo anterior, sobre todo a la última lógica de la identidad, se erigen los procesos de subjetivación que experimentan los entrevistados. Los desgarros en primera persona estarían dados por experiencias ligadas al servicio de salud, así como un distanciamiento crítico de la política institucional. De ello se desprende el deseo de ser actor, pero no cualquier actor sino que uno en específico: el actor colectivo de los movimientos sociales. Pero también cabe mencionar la presencia de desgarros dado por el sufrimiento de un otro, que genera indignación en estas personas. 
Así, en el proceso de articulación de estos actores, el problema de la salud toma una significación política, en la medida en que la demanda se levanta en contra de un adversario político explícito: el Estado de Chile, que avala el modelo neoliberal. La irrupción de este modelo en áreas que deberían ser derechos garantizados para todos, acompañado de un diseño con falta de visión de territorialidad es la principal queja presente en los discursos de estas personas. Sin embargo, se evidencia la presencia de un proceso de desidentificación propio de la subjetivación política, en la medida en que se asume que el problema no es de Chiloé en particular, sino que de todo el país, donde Chiloé es uno más. De esta manera, surge lo que se denomina una orientación a la totalidad, es decir, un proyecto de sociedad no acabado, pero que transciende el problema de la salud en su contingencia. Por todo esto, y en virtud de su articulación, estas personas generan acciones y prácticas de resistencia en dos sentidos: exógenos, en la medida que visibilizan demandas e incluso, intentan disputar el centro político con una candidatura independiente a diputado. Pero también es posible identificar prácticas de resistencia hacia el interior de la organización: la recuperación de valores propios como son la solidaridad, el cooperativismo y la vida en comunidad.

En base a todo lo anterior, cabe establecer la relación que toma el proceso de construcción de identidad y el de subjetivación política en las personas que participan del movimiento por la salud, respondiendo a nuestra pregunta de investigación. Como pudimos ver, los procesos de subjetivación están íntimamente relacionados con la identidad de estas personas, en la medida en que la identidad se entiende como el movimiento entre una afirmación negativa: la experiencia nostálgica por un pasado perdido, asociado a valores compartidos, y por una afirmación positiva: la promesa o el compromiso, íntimamente ligado al deseo de ser actor colectivo, propio de la subjetivación. Pero vimos además que la subjetivación es en sí misma un proceso de desidentificación y consiguiente reidentificación, un ejercicio de carácter político, dado por entender que el problema de la salud no es propio del archipiélago, ni tampoco individual, sino que colectivo y generalizado a nivel país, asociado a un adversario político que en este caso es el Estado chileno.

En este sentido, la hipótesis inicial que guió esta investigación, a saber: la subjetivación política de estas personas se construye en relación a lo que se puede denominar un vínculo "dañado" entre los individuos de esta zona con el Estado a través de las políticas de salud, pero, a su vez, asociado a un fuerte componente identitario, en términos de arraigo, no es corroborada del todo, en la medida en que se puede constatar la presencia de un fenómeno mucho más complejo en términos de identidad y subjetivación política. La identidad no sería una afirmación positiva ni esencialista dada por un arraigo, sino que la articulación de lógicas de acción no unitarias, con variantes positivas y negativas, donde el Estado además es un referente más dentro de una serie de desgarros de la biografía, en lo que se entiende por subjetivación política. 
Por otro lado, llama la atención el nivel de politización y la forma que toma la subjetividad política en las personas entrevistadas. A modo de hipótesis emergente, dicha configuración se puede explicar producto de que, a pesar de que estas personas corresponden a sectores medios, las condiciones de precariedad en términos de salud pública y otros derechos básicos a las que están expuestos cotidianamente son tales, que parecen tener mayor correspondencia a las que vivenlas clases populares. En directa relación con los estudios de subjetividad popular (Angelcos 2010) donde la subjetividad política de las personas estaría marcada por procesos de exclusión social y pobreza, las experiencias recién expuestas no parecen distar mucho de dicha realidad en torno al problema de salud. Así, se puede establecer que la posición de clase media aparece sobredeterminada por el carácter provincial e insular de Chiloé, lo que la acerca, en términos de experiencia social, a la vulnerabilidad que caracteriza a los sectores populares.

Sin embargo, la limitación en términos de tiempo impide hacer tal afirmación. Queda por tanto, como desafío pendiente la profundización de la dimensión de clase en los estudios a futuro sobre subjetividad.

Ahora bien, cabe mencionar nuevamente que las conclusiones esbozadas corresponden al estudio de casos de asambleas de Ancud y Castro, donde la posibilidad de hacerlas extensivas a todo el movimiento por la salud en Chiloé son limitadas, en la medida en que la realidad social de estas comunas es muy diferentes a lo que sucede en el resto del archipiélago, especialmente en Quellón. Queda como desafío a futuro conocer el caso de dicha comuna, lugar de surgimiento del movimiento por la salud digna en Chiloé.

En virtud de todo lo anterior, cabe destacar la importancia del estudio de los procesos de subjetivacióna la hora de comprender de mejor manera los procesos de acción colectiva y luchas sociales, presentes en nuestra sociedad cada vez con mayor fuerza. 
Polis, Revista Latinoamericana, Volumen 15, $N^{\circ}$ 44, 2016

\section{Notas}

${ }^{1}$ Este artículo resume los resultados de la investigación realizada en el marco del Seminario de Grado para optar al título de sociólogo(a), Universidad Alberto Hurtado, aprobada el año 2014, y premiada en el marco del concurso "Mejores seminarios de grado 2012-2013”, Departamento de Sociología, de la misma casa de estudios.

${ }^{2}$ Coalición de partidos políticos de centro, centroizquierda e izquierda, que gobernó el país durante el periodo 1990-2010.

${ }^{3}$ Población para el año 2015 según proyecciones poblaciones INE: http://www.ine.cl/ canales/chile_estadistico/familias/demograficas_vitales.php

${ }^{4}$ La Minga significa, acorde con la RAE: Reunión de amigos y vecinos para hacer algún trabajo gratuito en común.

${ }^{5}$ Cabe mencionar que hablamos de "adversario político" y no social al referirnos al principio de oposición en la presente investigación, ya que el choque entre adversarios no es entre clases sociales, sino que entre el movimiento social y el Estado de Chile.

${ }^{6}$ Se habla de "orientación a un principio de totalidad” y no "totalidad” propiamente tal, ya que es posible dilucidar cierta idea de sociedad más allá del problema de salud, más no un proyecto propio de sociedad, que es a lo que hace referencia este principio. 


\section{Bibliografía}

Angelcos, Nicolás (2010), “La estructuración de la subjetividad popular y el problema de la política”. Revista de Psicología, Universidad de Chile. Vol. 19, No 2, Santiago de Chile.

Ídem (2008), Subjetividad y política. Sobre el rendimiento sociológico de los procesos de subjetivación. Memoria para optar al título de sociólogo. Universidad de Chile, Santiago de Chile.

Azócar, Carlos (2012), “Lo público y lo privado, la educación y los movimientos sociales en un contexto neoliberal chileno. Prácticas, aprendizajes y limitaciones de la construcción de una nueva ciudadanía”. Anuari del Conflicte social 2012, Barcelona.

Martuccelli, D. y Singly de F. (2012), Las sociologías del individuo. Lom ediciones, Santiago de Chile.

Dubet, Francois (2010), La sociología de la experiencia. Ed. Complutense, Madrid.

Ídem (1989), “De la sociología de la identidad a la sociología del sujeto”. En Revista Estudios Sociológicos, vol. VII, núm.21. El Colegio de México.

Durán, Carlos (2012), El acontecimiento estudiantil y el viraje del proceso sociopolítico chileno. OSAL (CLACSO). Año XII, n 31. Buenos Aires.

Espinoza, Vicente (1993), Pobladores, participación social y ciudadanía. Proposiciones, $\mathrm{n}^{\circ} 22$. Ediciones SUR, Santiago de Chile.

Fernández, Juan (2013), “Movimiento estudiantil en Chile 2011: repertorios de acción, marcos de acción colectiva, impactos para la política pública”. Circunstancia, año XI, n 31.

Fleet, Nicolás (2011), “Movimiento estudiantil y transformaciones sociales en Chile: una perspectiva sociológica”. Revista Polis, vol. 10, n 30 . Santiago de Chile.

Garretón, M. et al (2011), “Movimiento social, nuevas formas de hacer política y enclaves autoritarios. Los debates del Consejo Asesor para la Educación en el gobierno de Michelle Bachelet”. Revista Polis, vol.10. n 30. Santiago de Chile.

Honneth, Axel (1997), La lucha por el reconocimiento. Por una gramática moral de los conflictos sociales. Editorial Crítica, Barcelona.

Larraín, Jorge (2001), Identidad chilena. LOM ediciones, Santiago de Chile. 
Mayol, A. \& Azocar, Carla (2011), “Politización del malestar, movilización social y transformación ideológica: el caso de Chile 2011 ”. Revista Polis, vol. 10, $\mathrm{n}^{\circ}$ 30. Santiago de Chile.

Mayol, A., Azocar, C. \& Brega, C. (2011), El clivaje público/privado: el horizonte último del impacto del movimiento estudiantil en Chile 2011. Santiago de Chile.

Mira, Andrea (2011), “Crisis de representatividad y estallido social. Una aproximación a la actual experiencia chilena”. Revista Polis, vol. 10, n 30. Santiago de Chile.

Montecinos, Egon (2013), “Los actuales desafíos regionales en Chile: ¿Nueva regionalización o mayor descentralización?” Universidad de los Lagos. Centro de estudios desarrollo regional y local- CEDER.

Montecinos, E. \& Neira, V. (2012), “Gobernanza democrática y multinivel en la intervención territorial: el caso del plan Chiloé en Chile”. En Delamaza, G., Cunill, N. y Joignant, A. (Eds): Nueva agenda de descentralización en Chile. RIL editores, Santiago de Chile.

Mondrego, F., Ramírez, E., Macé, J., Yañez, R. (2009), Caracterización de los actores del Chiloé Central. Documento de trabajo $n^{\circ} 55$. RIMISPCentro latinoamericano para el desarrollo rural.

Núñez, Daniel (2012), “Proyecciones políticas del movimiento social por la educación en Chile”. En OSAL (CLACSO) año XII, n 31 . Buenos Aires.

Rancière, Jacques (2006), Política, Policía y Democracia. LOM ediciones. Santiago de Chile.

Segovia, R. \& Gamboa, C. (2012), “Chile: el año en que salimos a la calle”. Revista de Ciencia política, vol. 30, n¹. Santiago de Chile.

Taylor, Charles (1997), La política del reconocimiento. Argumentos filosóficos. Paidos, Barcelona.

Touraine, Alain (1994), Crítica a la modernidad. Fondo de cultura económica, Buenos Aires.

Ídem (1995), La producción de la sociedad. UNAM, IFAL, México.

Ídem (1994), Crítica de la modernidad. Fondo de Cultura, México.

Urra, Juan (2012), “La movilización chilena estudiantil en 2011”. En OSAL (CLACSO) año XII, n³1. Buenos Aires. 
Valenzuela, Esteban (1999), Alegato histórico regionalista. Ediciones Sur, Santiago de Chile.

Recibido: 30.03.15

Aceptado: 21.09.15 